\title{
Research on the four wheel hub motor control system of the electric vehicle based on DSP
}

\author{
Peicheng Shi ${ }^{a}$, Jun Zhang, Rongyun Zhang and Shanshan Peng \\ Anhui Polytechnic University, New Vehicle Technology Research Center of Anhui Province, 241000Wuhu, China
}

\begin{abstract}
This paper mainly solves the problem of the independent driving and coordinated control of the four wheel motor in electric vehicle. The vehicle controller is designed based on DSP2407, the DA_OUT function is added to the program of the DSP2407 SPI module, which can be used to set the parameters SPI_DATA according to the corresponding relationship between the digital quantity of sensors and the speed of the four wheels. The four channels can be programmed independently. From the experiment figures, it can be known that the method proposed in this paper can realize the independent driving of the four wheels motor, which provides the basis for the future research of the electric vehicle turning and other complex working conditions.
\end{abstract}

Keywords: hub drive; electric vehicle; DSP controller; hub motor control.

\section{Introduction}

The key research of the pure electric vehicles is mainly composed of the three parts, including the motor drive control technology, the vehicle control and management and the energy recovery management system, the motor drive control technology is the heart of the pure electric vehicles[1].

The hub motor drive form is called by the industry as the ultimate driving form of the electric vehicles. The four wheel drive torque can be controlled independently, and the torque and speed can also be measured easily, so compared the existing traditional electric vehicle (only a concentrated large motor drive mode), in the aspect of the stability, active safety control and energy saving, the hub motor drive electric vehicle has a significant advantage, which is also the ideal carrier of the new generation of the vehicle control technology and exploring the vehicle dynamics performance, and has become one of the world's major automobile companies and research institutes research hot[2].

The four brushless DC motors of the wheel drive electric vehicle can be embed directly into four wheels, and the driving force is derived from four wheel hub motors. Through the DSP vehicle controller AD module, the holzer accelerator pedal and steering wheel angle signal can be gathered, after the precise calculation and independently programming, the four analog signal can be transmitted to the four in-wheel motor by using the SPI module, which can make the four in-wheel motors rotate at different or same speed according to the driver's desired conditions, so as to achieve the control of the four wheel drive motor.

\footnotetext{
${ }^{\text {a } C o r r e s p o n d i n g ~ a u t h o r ~: ~ s h i p e i c h e n g @ 126 . c o m ~}$
} 


\section{Four wheel hub motor control system}

Brushless DC motor is characterized by small volume, light weight, high efficiency and energy saving, good control and the electromagnetic torque ripple, etc. it has been widely used in the electric vehicle because of the above advantages[3]. The in-wheel motor studied in this paper is the brushless DC motor. According to the motor rotor position signal collected by hall position sensor, PWM wave is send by the motor driver to the full bridge inverter, in order to control the on-off time of the six power tubes (T1-T6) to achieve a brushless DC motor speed control[4]. The experiment in this paper mainly uses the DSP2407 to collect the acceleration pedal signal to control four brushless DC motor drivers and then realize the speed regulation of the four brushless DC motors. The connection schematic diagram of the brushless DC motor and the full bridge inverter driver is shown in figure 1(a). The physical map is shown in figure 1(b).

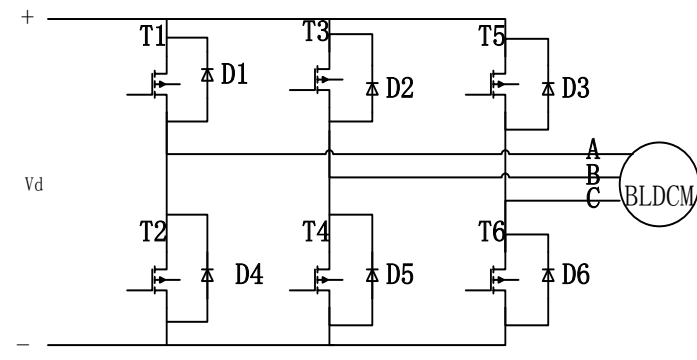

(a)

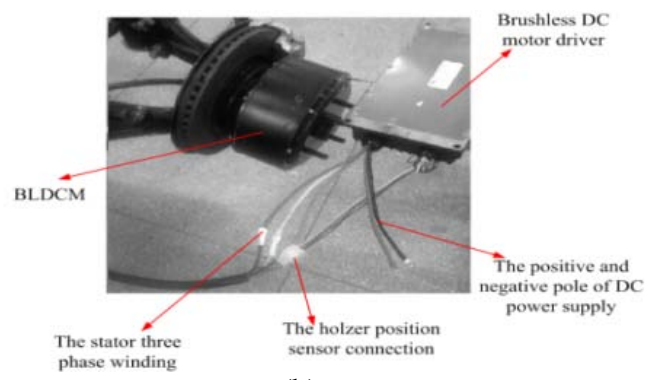

(b)

Figure 1. (a) Schematic diagram of BLDCM and full bridge inverter, (b) Physical map of BLDC and full bridge inverter

The DC power supplied to the full bridge inverter is the six $12 \mathrm{~V}$ lead acid batteries connected in series, and the PQ165 type foot holzer accelerator is used to gather the accelerator pedal signal. The TMS320LF2407 which is belong to the TI company is taken as the DSP controller. The block diagram of the whole four wheel hub motor control system is shown in figure 2, and the physical map is shown in figure 3(a).

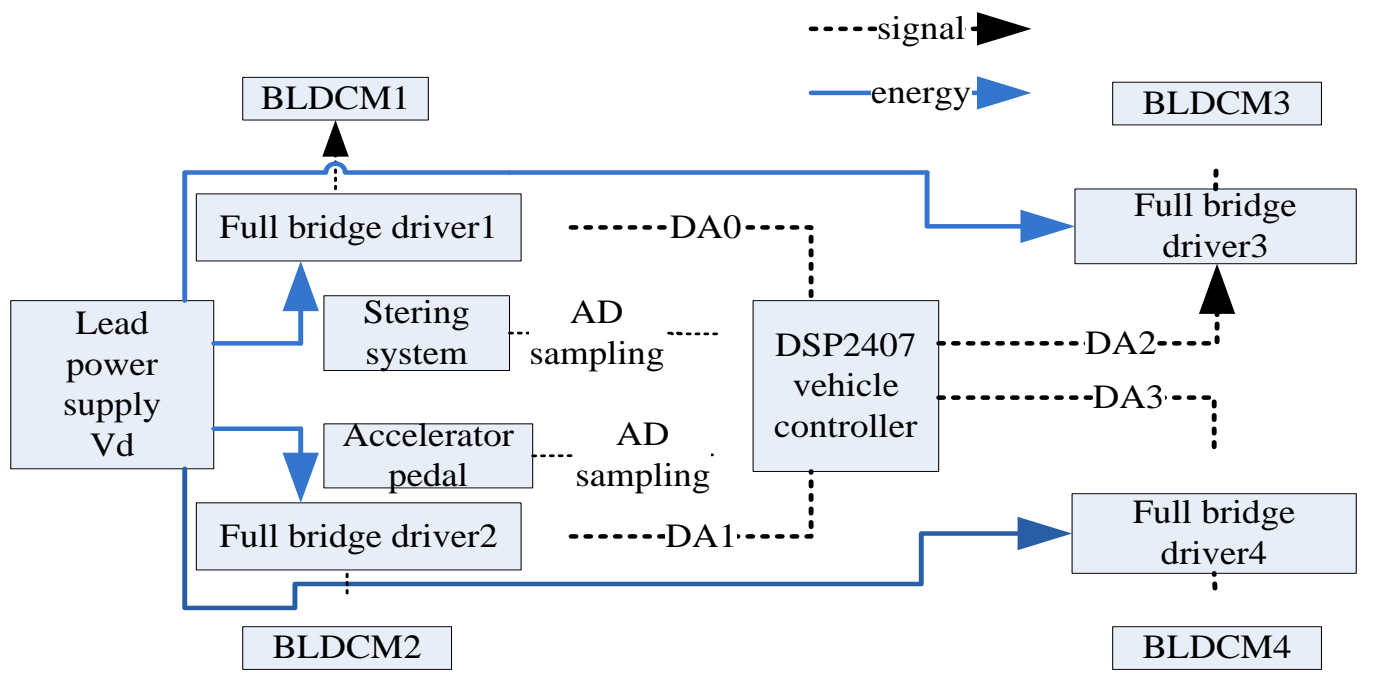

Figure 2. Four wheel hub motor control system 


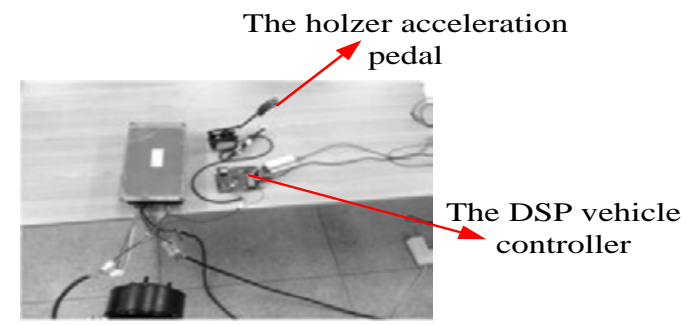

(a)

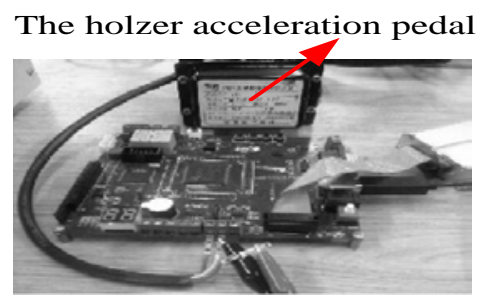

(b)

Figure 3. (a) Physical map of four wheel hub motor control system, (b) Connection diagram of accelerator and DSP

\section{AD sampling of the acceleration pedal output signal}

The 10 bit ADC with sample and hold function, up to 16 channels of analog input channels (ADCIN0ADCIN15), and two independent sorting devices with up to 8 analog conversion channels(SEQ1 and SEQ2) are built in the DSP2407[5]. In order to complete the test, the output signal of the holzer acceleration pedal, a channel analog input(ADCIN0) which does not need continuous conversion, and maximum conversion channels register $(\mathrm{Max} \mathrm{CONV}=$ channel -1$)$ need be used. When CONT RUN=0, the ADC is enabled to work in the start/stop mode. Select sort SEQ1, software startup ADC.

Due to the DSP2407 AD converted is a 10 bit, the digital quantity after conversion is deposited in the left of results register RESULT0, so the AD conversion results need right shift six units to ensure the accuracy of the conversion results. In addition, in order to ensure an ad conversion is complete at once time, it is need to add an waiting instructions whether the query SEQ1 BSY bit is 0, which will not be occur that the results of the first sample put into the next result register and then leads to the AD sampling digital quantity does not match to the input analog quantity. According to the analysis of above, the AD DSP sampling function procedures are as follows:

void AD_Sample( )

\{ ADCTRL1=0x4000; $\quad \operatorname{asm}(" \quad$ NOP"); $\quad$ ADCTRL1=0x0020; $\quad$ MAXCONV=0x0000; CHSELSEQ1=0x0000; ADCTRL2=0x4000; ADCTRL2=0x2000; while( (ADCTRL2\&0x1000)==0x1000);asm(" NOP "); asm(" NOP ");RESULT_0=RESULT0>>6; \}

In the procedures, interrupt to enter the $\mathrm{AD}$ sampling is set to timer 1 , and every $100 \mathrm{~ms}$ to carry out a $\mathrm{AD}$ sampling, that is, the frequency is the ten times a second, because the frequency of a driver stepped on the accelerator pedal will not exceed 10 times/s. on the same channel the DSP2407 ADC module can sample for more several times, that is, for a certain channel, over sampling can be implemented, so the results of the sampling is higher accurate than the traditional AD sampling results. A AD sampling is carried out by the procedure of the test every $100 \mathrm{~ms}$, and in the main function, 18 times "over sampling" are carried out by the channel ADCIN0 to ensure the accuracy of the conversion results. The 18 conversion results are stored in the array AD0[], the DSP main function of the program as follows:

main()

\{ SystemInit(); Timer1Init(); asm(" CLRC INTM ");

while(1)// Waiting for the timer interrupt to make the AD_FLAG set 1

$\left\{\operatorname{if}\left(A D \_F L A G==1\right)\{\right.$ AD_FLAG $=0$; for $(\mathrm{i}=0 ; \mathrm{i}<18 ; \mathrm{i}++)$ //Sampling 18 times

\{ AD_Sample(); AD0[i]=RESULT_0; \} \} \} \}

The physical map of the connection of the holzer accelerator pedal and DSP vehicle controller is shown as follows, in figure3(b).

The actual output of the holzer accelerator pedal PQ165 is $0.8-3.2 \mathrm{~V}$, while the largest input voltage of DSP2407 is 3.3V, which can be directly input to the DSP2407 ADCIN0 channel without voltage conversion. When the holzer accelerator pedal is at initial state, the output voltage is $0.818 \mathrm{~V}$, as shown in figure $4(\mathrm{a})$. 


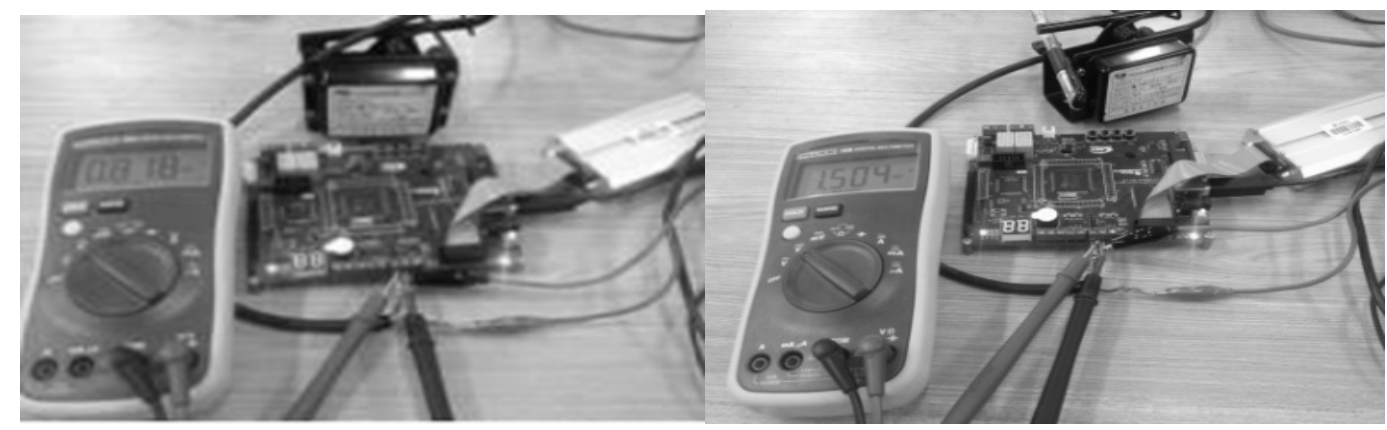

(a)

(b)

Figure 4. (a) Output voltage signal of the initial state of the holzer accelerator, (b) Output voltage signal of the pressure $8 \mathrm{~mm}$ in the holzer accelerator

The JTAG on-line debugging is carried out by the compiler CCS3.3. Use the Watch Window function of the compiler CCS3.3 to view the results of the AD conversion array AD0[] values.

From the Watch Window function of the compiler CCS3.3, we can see that the 18 conversion values are around the 246, the full range of the input is 3.3V, the DSP2407 ADC is 10 bit,the input analog quantity corresponding to the digital quantity 246 is as follows:

$$
\frac{246}{2^{10}} \times 3.3 V \approx 0.793 \mathrm{~V}
$$

Compared with the actual analog input of holzer accelerator pedal, the error is $0.025 \mathrm{~V}$, which is fully satisfied with the accuracy requirements. In order to further verify the accuracy of the AD conversion, Pressing holzer accelerator pedal about a pen depth (about $8 \mathrm{~mm}$ ), the output voltage is 1.504, the results of the experiment as shown in figure4(b).

And from the Watch Window function of the compiler CCS3.3, the digital value converted is about 465 , the same as the initial state of the calculation process, the analog quantity corresponding to the digital quantity is:

$$
\frac{465}{2^{10}} \times 3.3 \mathrm{~V}=1.499 \mathrm{~V}
$$

The error with the actual holzer accelerator pedal is $0.005 \mathrm{~V}$. At this point, the output signal of the holzer accelerator pedal is collected precisely by DSP.

\section{SPI output four channel analog signal control four wheel motor}

DSP2407 includes a serial peripheral interface SPI module, SPI is a high-speed, synchronous serial I/O port, which allows the length of programmable serial bit stream (1-16 bit) to move in or out of the device with programmable bit transmission speeds.Usually SPI is used for the communication between the DSP processor and the external peripherals as well as other processors ${ }^{[6]}$.

After AD sampling successful, according to the digital quantity after AD conversion, DSP through the SPI sent out four road analog signals that is the same with the output value of the holzer acceleration pedal, through the DA ports of the DSP vehicle controller, the four road analog signals are transmitted to the four brushless DC motor drivers. Then according to the size of the four input analog quantities, the brushless DC motor driver changes the size of the PWM duty cycle, so as to achieve the four wheel motor speed control.

The SPI serial D/A conversion chip of the test used is TLV5620, LV5620 is a four channel 8 bit voltage output D/A converter with a high impedance buffered input. its connection with the DSP as shown in figure 5 . 


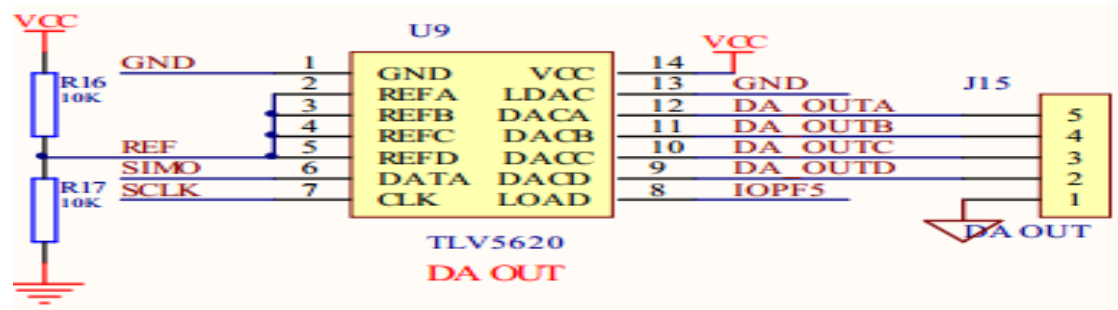

Figure 5. Connection diagram of TLV5620 and DSP

As is shown in figure5, the reference voltage input to the TLV5620 is half of 3.3V the DSP supplied voltage, which is $1.65 \mathrm{~V}$. And the full range of the output signal of the holzer accelerator pedal is $3.2 \mathrm{~V}$, so the SPI program designed must be that the output voltage range of TLV5620 is 1 times than the reference voltage.

The working frequency of TLV5620 is $1 \mathrm{MHz}$, the baud rate of SPI should be set to. From figure 5 we can see that the serial port of TLV5620 is connected to the IOPF5 port of DSP, the IOPF5 pin must be set to the general I/O port and the output mode, the transmission data length is 8bit, the SPI initialization procedures are as follows:

void SPI_Init()

$\{$ MCRB=MCRB | 0x0014; MCRC=MCRC \& 0xDF00;PFDATDIR=PFDATDIR 0x2020;SPICCR=0x004a; SPICTL=0x0006;SPIBRR=0x0027;SPICCR=SPICCR | 0x80; \}

The multiple form parameter RNG in D/A output function is very important, which directly determines the range of DA port output analog quantity. The formal parameter SPI_DATA is the digital quantity of DA needed to be converted, and its size is closely related to the value of the AD sampling result register. So SPI_DATA is the key to realize the four way analog signals which can be programmed independently, between which and the value of the AD sampling result register the relationship directly determines the speed of the four wheels.

In order to realize the continuous output analog quantity, it is also required to add virtual read register instructions to clear the interrupt flag. DA output function is as follows:

VoidDA_OUT(unsigned CHANNEL,unsigned int RNG,unsigned int SPI_DATA)

$\{$ unsigned char flag $=0$;SPITXBUF $=($ CHANNEL $<<14) \mid($ RNG $<<13) \mid($ SPI_DATA $<<5)$;

while(1) $\{$ flag=SPISTS\&0x40; if(flag $==0 \times 40)$ break; $\}$

SPIRXBUF=SPIRXBUF;PFDATDIR=PFDATDIR \& 0xFFDF;

for(i=0;i<5;i++);PFDATDIR=PFDATDIR | 0x0020; \}

The value of AD sampling result register is a 10bit, but DA can only deal with the 8-bit data, so the holzer acceleration pedal AD sampling result register value must be right shifted two position, that is, divided by 4 and then sent to DA_OUT function formal parameter SPI_DATA, which can be realized, as long as the following codes are added after the AD sampling function AD_Sample().

SPIDATA=AD0[i] $>>2 ; D A \_O U T(0,1$, SPIDATA);/RNG=1

DA_OUT(1,1,SPIDATA);DA_OUT(2,1,SPIDATA);DA_OUT(3,1,SPIDATA);

In the initial state of the holzer accelerator pedal, the four way DA port output analog results measured by digital multimeter as shown in figure 6 .

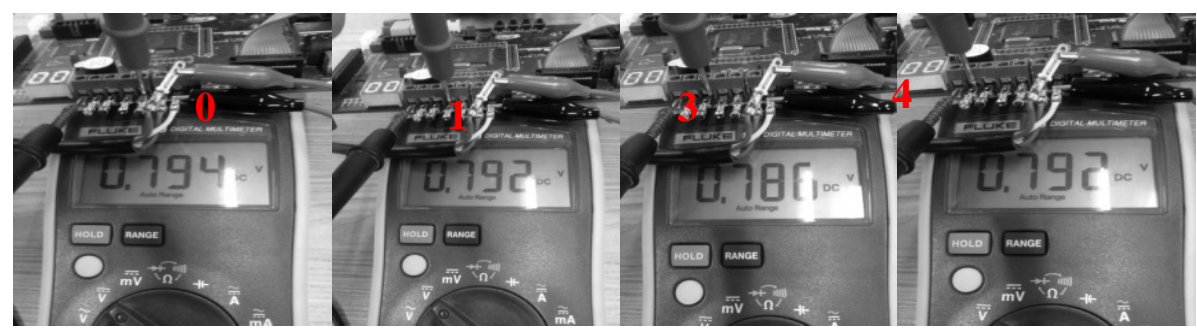

Figure 6. Four DA port output analog value 
From figure 6 we can see that the four analog signal output is consistent, and the error is in a few millivolts. Here we only need to take one way to verify the DA output analog signal and holzer accelerator output signal is consistent. The waveform of the output signal of DA_OUT0 and holzer accelerator is shown in Figure 7.

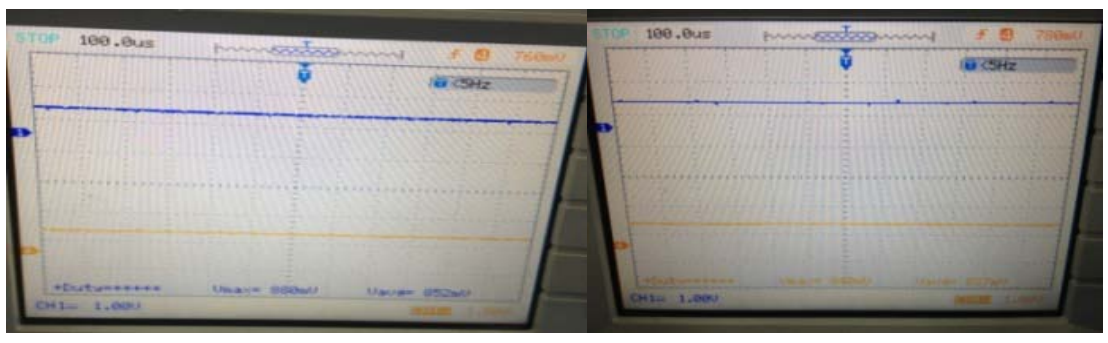

Figure 7. Waveform of the output signal of DA_OUT0 and holzer accelerator

In the figure 7 , the output signal of the holzer accelerator pedal is input from the digital oscilloscope channel 1 , the waveform is blue, the average voltage is $852 \mathrm{mV}$. The output signal of DSP2407 DA_OUT0 is input from the digital oscilloscope channel 4, waveform is yellow, the average voltage is $827 \mathrm{mV}$. The error is $25 \mathrm{mV}$, and very small, which indicates that the output of DA_OUT0 is basically the same as that of the holzer accelerator pedal output signal. thus, according to the output signal of the holzer accelerator pedal gathered by AD sampling, DSP vehicle controller through the SPI successfully sent out the four way analog signals which can be individually programmed.

TheDSP2407 sends 4 channel analog signal commands to the four brushless DC motor drivers, changing the duty cycle of the PWM issued by the driver to achieve the wheel motor speed control, and then to achieve the desired car running conditions.

\section{Conclusion}

In this paper, the TI company's DSP TMS320LF2407 is used as the core of the vehicle controller, the supplied voltage of DSP2407 is 3.3V, which can reduce the consumption of the whole vehicle controller, this is very favorable to the electric vehicle energy saving. The execution speed of 30MIPS improved the real-time performance of the whole vehicle controller .

The results of this study provide a basis for the future research of electric vehicle turning and complex road conditions. For example, the turning of the vehicle is a vehicle controller based on the steering wheel sensor gathered by the AD sampling sent out the four way analog signals to change the wheel motors speed, which can be realized by getting the relationship between the steering wheel angle value and the each motor speed.

\section{Acknowledgments}

The authors thank that this research is supported by the National Natural Science Foundation of China (GrantNo. 51575001) and Anhui province science and technology research key project (Grant No. 1604a0902158) and Anhui university scientific research platform innovation team building projects(2016-2018).

\section{References}

1. S. Q. Tang, D, X. Liao, Z. J. Wu. Key technology and prospects on the electric vehicles. Mech.Sci. Technol., 22 (2): 189-192 (2003)

2. R. He, R. J. Zhang. Research and development of in-wheel motor drive technology.J. Chongqing Univ. Technol.,29 (7): 10-18 (2015) 
3. F. Cui, F. G. Liu, Z. H. Qiu, Y. P. Wei. Design of controller of Brushless DC motor for electric vehicle based on DSP. Electr. Mach. control appl., 41(4): 52-56 (2014)

4. C. L. Xia, H. W. Fang. Permanent-magnet brushless DC motor and its control. T. of China Electrotech. Soc., 27(3): 25-34 (2012)

5. Y. P. Yi, D. Li, F. Xiao. Design of the signal detection and conditioning circuit based on the DSP chip A/D. inform.Technol., 20(1):5-8 (2013)

6. K. T. Zhang, J. H. Yang, X. H. Du. The design and implementation of serial communication based on DSP. micro process., 6:23-26 (2010) 